Discussion Paper $\sharp 1998-12$

EFFICIENCY OF STOCHASTIC TRANSFERS

IN A DIRECTED GRAPH

by

Akira YAMAZAKI

February, 1999 


\title{
Efficiency of Stochastic Transfers in a Directed Graph
}

\author{
Akira Yamazaki \\ Graduate School of Economics \\ Hitotsubashi University \\ Kunitachi, Tokyo 186-8601, Japan
}

\begin{abstract}
A mathematical model of a directed graph with stochastic transfers is presented. It will be used to analyze the optimality (or "competitiveness") properties of a network of transactions involving risky transfers of assets in an economic system. These properties are discussed in a model with some specific directed graph structures which result in a decompositon of the graph into parts with "narrow" linkage.
\end{abstract}

\section{Introduction}

The purpose of this paper is to present a mathematical model of a directed graph with stochastic transfers, which will be used to analyze the optimality (or "competitiveness") properties of a network of transactions involving risky transfers of assets in an economic system.

A mathematical model of stochastic transfers in a directed graph is presented in section 2. The concepts of optimality of stochastic transfers are discussed in terms of efficiency and competitiveness in section 3. A specific model of risky transfers of assets, which will be called the "Wicksell triangle" plus a simple barter is presented in section 4. Optimality properties of risky transfers in the specific model are briefly discussed in section 4 .

\section{A Model of Stochastic Transfers in a Directed Graph}

\section{$2.1 \quad$ A directed graph}

An ordered pair $(A, \mathcal{T})$ consisting of a nonempty finite set $A$ and a binary relation $\mathcal{T}$ on $A$ is a directed graph. The interpretation of the graph $(A, \mathcal{T})$ in this paper is the following: 


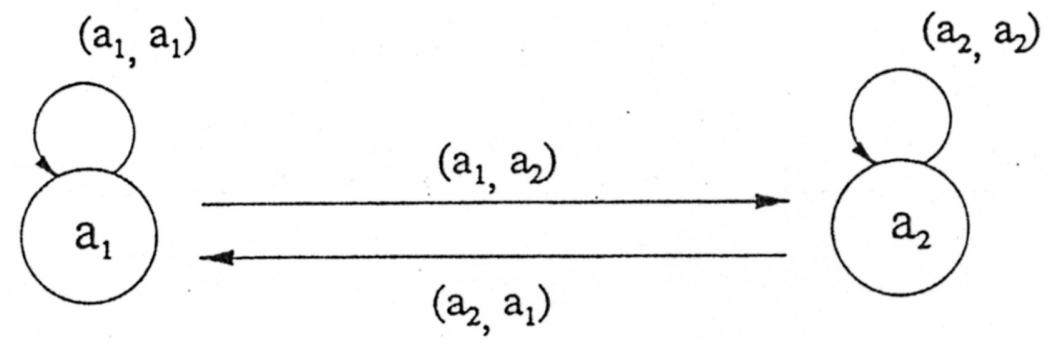

A "Simple Barter"

Fig. 1

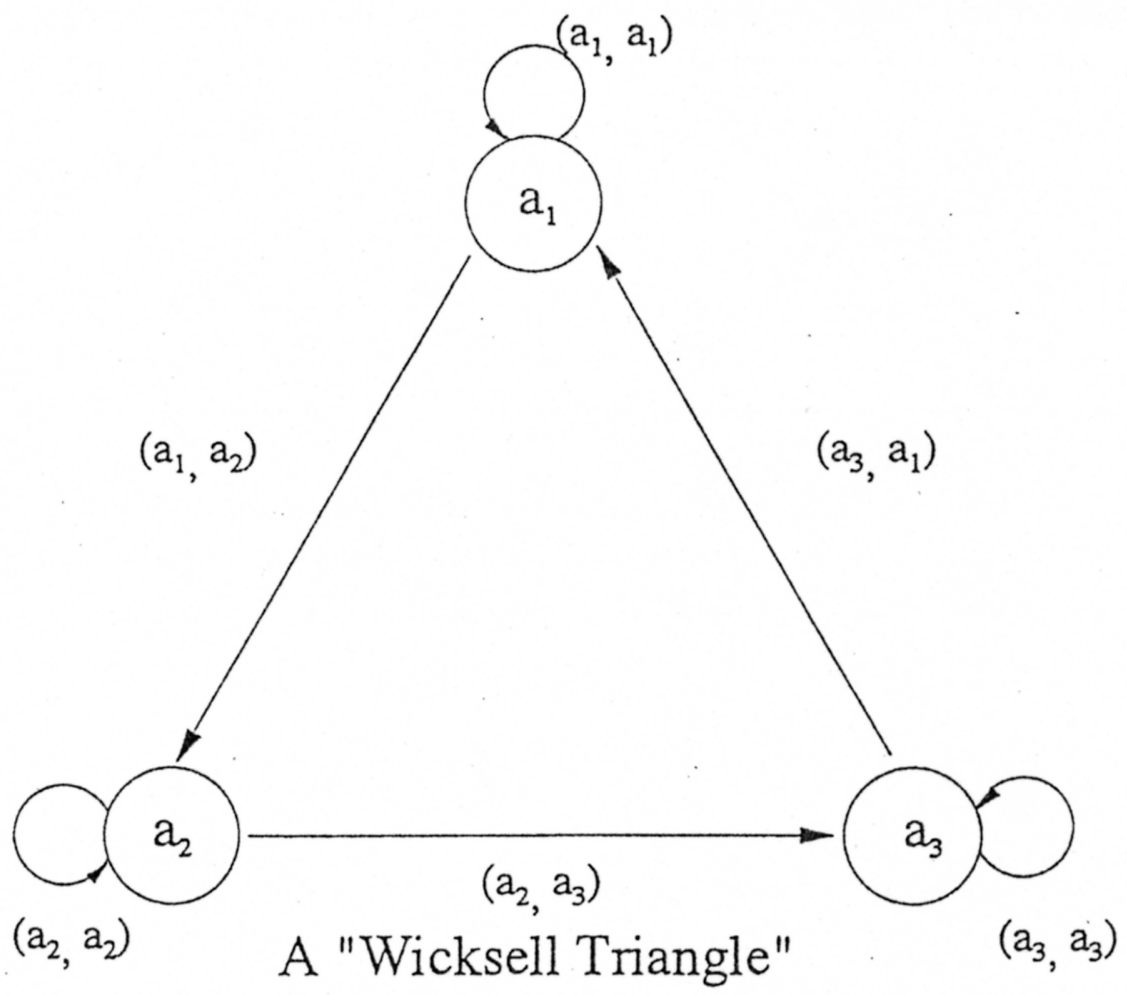

Fig. 2 
a point $a \in A$ is a trader or an agent; an ordered pair $\left(t_{1}, t_{2}\right) \in \mathcal{T}$ shows a direction of transfer, i.e., a transfer from $t_{1}$ to $t_{2}$.

Two typical examples of a directed graph arising from commodity and financial assets transfers are illustrated in figure 1 and figure 2. Figure 1 illustrates a simple barter trade transfers of commodities or assets. Agent $a_{1}$ sends commodities or assets to agent $a_{2}$ and vice versa. Then, it is represented by a directed graph $(A, \mathcal{T})$ with $A=\left\{a_{1}, a_{2}\right\}$ and $\mathcal{T}=\left\{\left(a_{1}, a_{2}\right),\left(a_{2}, a_{1}\right),\left(a_{1}, a_{1}\right),\left(a_{2}, a_{2}\right)\right\}$. In figure 2 , each agent sends commodities or assets counterclockwise to another agent, indicating the fact that there are no "double coincidence of wants." This type of transfers is known as a "Wicksell triangle." It is represented by $(A, \mathcal{T})$ with $A=\left\{a_{1}, a_{2}, a_{3}\right\}$ and $\mathcal{T}=\left\{\left(a_{1}, a_{2}\right),\left(a_{2}, a_{3}\right),\left(a_{3}, a_{1}\right),\left(a_{1}, a_{1}\right),\left(a_{2}, a_{2}\right),\left(a_{3}, a_{3}\right)\right\}$. Figure 3 gives an illustration of a general directed graph.

\subsection{Probability structure}

The stochastic transfer will be formalized in terms of a state space $\Omega$. We assume that a probability measure space $(\Omega, \operatorname{Pr})$ is given.

\subsection{Commodities, endowments and preferences}

Let there be $\ell$ kinds of commodities or assets. (Henceforth, assets and commodities are not distinguished and they are simply referred to as commodities.) $\mathbb{R}^{\ell}$ represents the space of these $\ell$ commodities. The initial endowments of each agent $a \in A$ of these commodities are specified by a given mapping $e: A \rightarrow \mathbb{R}_{+}^{\ell}$. Thus, each agent $a$ is endowed with the bundle of commodities $e(a) \in \mathbb{R}_{+}^{\ell}$.

In order to discuss state-contingent trade of these endowments, we adopt Arrow's convention that each type of commodity is a class of state-contingent commodities, one for each state in $\Omega$. Thus the set of all commodities is $\Omega \times\{1, \ldots, \ell\}$. Each agent $a$ is endowed with $e^{j}(a)$ units of commodity $(\omega, j)$ for every $\omega \in \Omega$. Formally, the commodity space $L$ is given by ${ }^{1}$

$$
L \equiv\left(\mathbb{R}^{\ell}\right)^{\Omega}
$$

A commodity bundle $x \in L$ may be viewed as a random vector defined on $\mathbb{R}^{\ell}$ or a measurable mapping $x$ defined on $\Omega$ taking values in $\mathbb{R}^{\ell}$.

We assume that each agent's preference between commodity bundles conforms to expected utility. Agent $a$ has a von-Neuman-Morgenstern utility function $u_{a}: \mathbb{R}^{\ell} \rightarrow \mathbb{R}$. Then,

\footnotetext{
${ }^{1}$ If one wants to conform to the Arrow-Debreu convention strictly, then one needs to distinguish commodities by each state, the origin and the place of its consumption. Then, the commodity space $L$ should be given by $L \equiv\left(\mathbb{R}^{\ell}\right)^{\Omega \times \mathcal{T}}$ where for $t=\left(t_{1}, t_{2}\right) \in \mathcal{T}, t_{1}$ shows the origin of the commodity whereas $t_{2}$ shows its place of consumption. In this paper we do not adopt the convention because we like to assume each agent derives utility from the consumption of commodities provided at his own location. This assumption not only simplifies our analysis but also helps us to focus on the effects of transfers on the final consumption attained by each agent.
} 


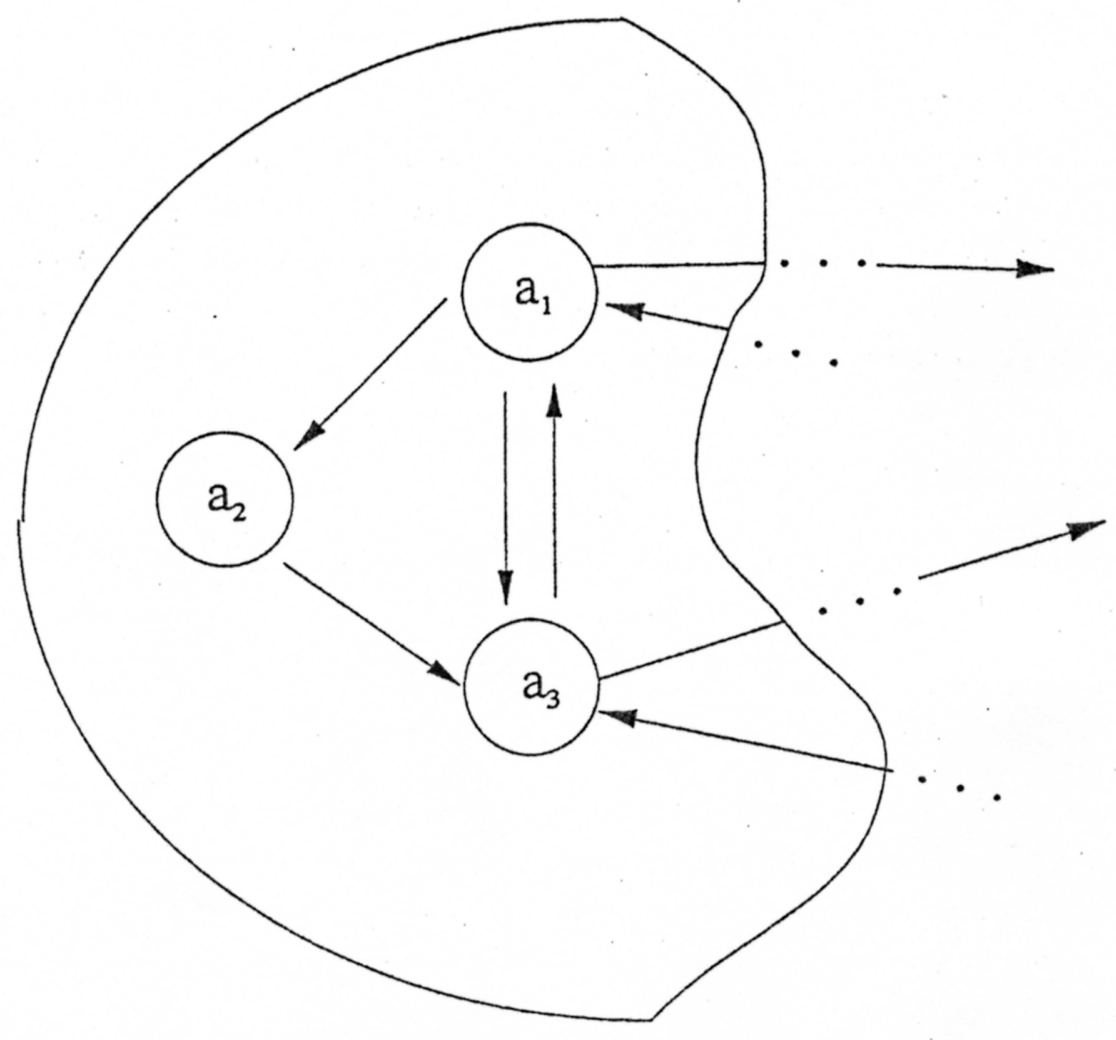

Fig. 3
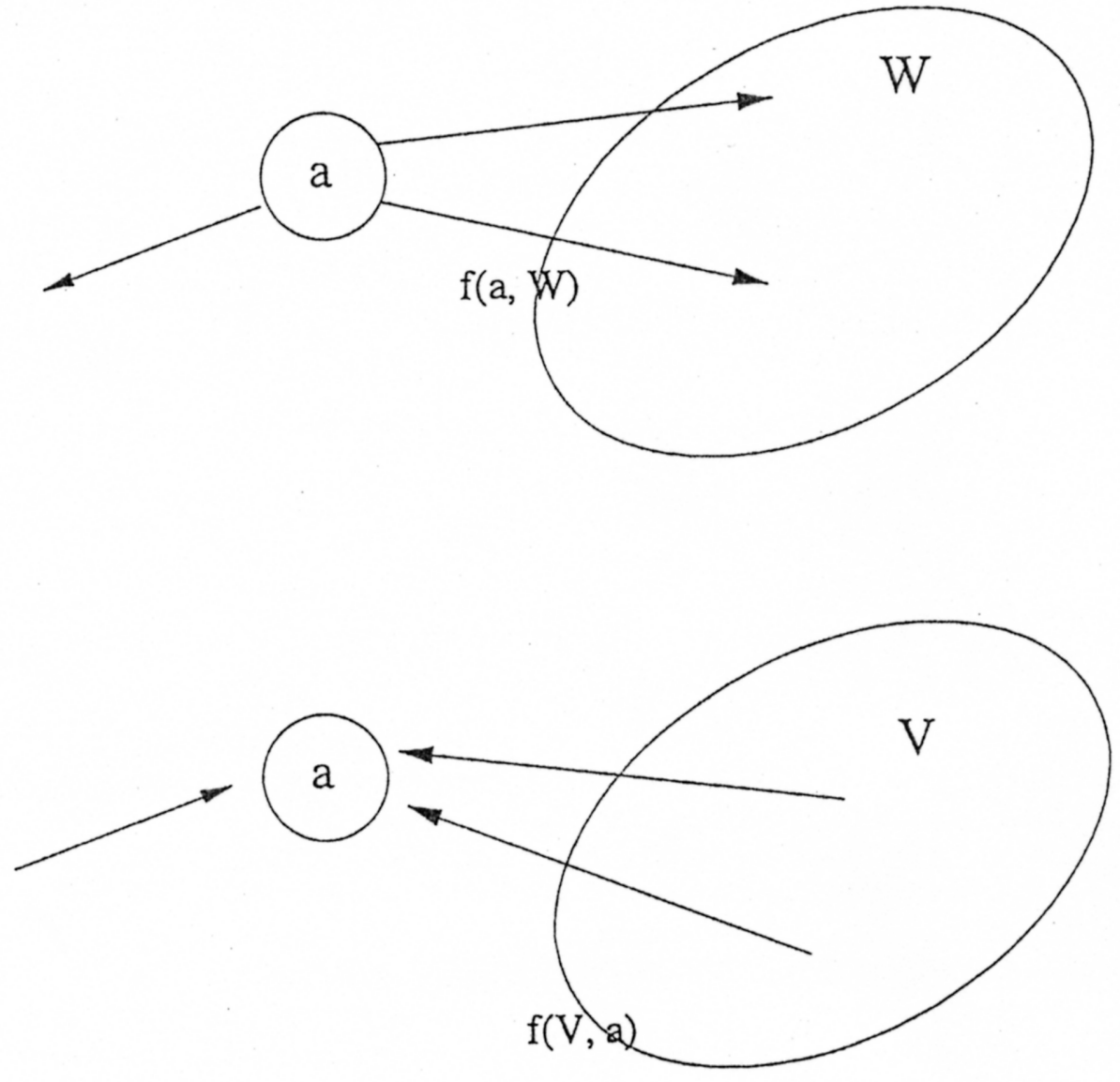

Fig. 4 
if $x \in L$ is a commodity bundle, the expected utility of $x$ for agent $a, U_{a}(x)$ is given by

$$
U_{a}(x)=\int_{\Omega} u_{a}(x(\omega)) d P r .
$$

\subsection{Information partition}

We shall consider two rounds of transfers. For each round of transfers, information of agents is specified by an information partition $\mathcal{P}$ on $\Omega$. Denote by $\mathcal{P}_{n}, n=1,2$, the partitions corresponding to these rounds. It is assumed that information becomes finer as rounds proceed so that $\mathcal{P}_{1} \subset \mathcal{P}_{2}$. In a specific model presented in section 4 , one assumes $\mathcal{P}_{1}=\{\Omega\}$ and $\mathcal{P}_{2}=\{S, F\}$. That is, there is no information available at round 1 and at round 2 information becomes available whether transfers have safely done, i.e., $\omega \in S$ or failed, i.e., $\omega \in F$.

\subsection{A network of stochastic transfers}

A given directed graph $(A, \mathcal{T})$ represents the infrastructure of a network of stochastic transfers. For the purpose of our present analysis, we consider two rounds of transfers. Each agent $a \in A$ in the network decides amounts and constituents of his transfer to other agents in the network at each round. We assume, however, that agents make an agreement for transfers of commodities among them before the initial round. The agreement among them is binding. The list of stochastic transfers among agents for each round of transfers is denoted by

$$
\tau_{n}: \Omega \times \mathcal{T} \rightarrow \mathbb{R}_{+}^{\ell}, n=1,2,
$$

where $\tau_{n}$ is $\mathcal{P}_{n}$-measurable, indicating that agents can use information available at the time of actual transfers. $\tau_{n}\left(\omega,\left(t_{1}, t_{2}\right)\right)$ represents a state-contingent transfer from agent $t_{1}$ to $t_{2}$ in state $\omega$ at $n$-th round.

The stochastic nature of rounds of transfers will be described by a stochastic safety rate function for each round, which indicates the safely arrived proportion of commodities that are sent from one agent to another. Stochastic safety rate functions are given by

$$
\sigma_{n}: \Omega \times \mathcal{T} \rightarrow[0,1], \quad n=1,2,
$$

where both $\sigma_{1}$ and $\sigma_{2}$ are $\mathcal{P}_{2}$-measurable. Since $\mathcal{P}_{1}$ is the information available at initial round, $\mathcal{P}_{2}$-measurability of $\sigma_{1}$ means that agents do not know for initial round transfers the actual proportion of commodities that reaches another agent, so that agents face risky transfers. On the other hand, $\mathcal{P}_{2}$-measurability of the stochastic safety rate function $\sigma_{2}$ for second round transfers means that agents exactly know the proportion of commodities that will reach another agent at the time transfers are to be made. Thus, agents can make second round transfer agreement contingent on the events in $\mathcal{P}_{2}$. When $0<\sigma_{2}<1$, it means that second round transfers are costly. This cost which is sometimes called as "iceberg cost," 
can be viewed as a crude way of reflecting various intuitive considerations including time preference and exposure to business loss due to delayed availability of transferred funds. We assume that the information $\mathcal{P}_{n}, n=1,2$, is publicly available at $n$-th round.

\begin{tabular}{|l||c|c|}
\hline Round & 1 & 2 \\
\hline \hline \multirow{2}{*}{ Transfer } & $\tau_{1}: \Omega \times \mathcal{T} \rightarrow L_{+}$ & $\tau_{2}: \Omega \times \mathcal{T} \rightarrow L_{+}$ \\
& $\mathcal{P}_{1}$-measurable & $\mathcal{P}_{2}$-measurable \\
\hline \multirow{2}{*}{ Stochastic Safety Rate } & $\sigma_{1}: \Omega \times \mathcal{T} \rightarrow[0,1]$ & $\sigma_{2}: \Omega \times \mathcal{T} \rightarrow[0,1]$ \\
& $\mathcal{P}_{2}$-measurable & $\mathcal{P}_{2}$-measurable \\
\hline
\end{tabular}

\subsection{A transfer network economy}

An ordered pair $\tau=\left(\tau_{1}, \tau_{2}\right)$ of stochastic transfer functions will be called a stochastic transfer. An ordered pair $\sigma=\left(\sigma_{1}, \sigma_{2}\right)$ of stochastic safety rate functions will be called a transfer technology.

A transfer network economy $\mathcal{E}$ will be given by a quintuple consisting of a directed graph $(A, \mathcal{T})$, an initial endowment function $e: A \rightarrow \mathbb{R}^{\ell}$, a specification of a utility function to each agent $\left(u_{a}\right)_{a \in A}$, a transfer technology $\sigma=\left(\sigma_{1}, \sigma_{2}\right)$, and an information structure $\mathcal{P}=\left\{\mathcal{P}_{n}\right\}_{n=1,2}$, i.e.,

$$
\mathcal{E}=\left\{(A, \mathcal{T}), e,\left(u_{a}\right)_{a \in A}, \sigma, \mathcal{P}\right\}
$$

Let us introduce some further notation for convenience. For any $f: \mathcal{T} \rightarrow R_{+}^{\ell}, V, W \subset A$, we write

$$
f(V, W)=\sum_{t \in U} f(t)
$$

where $U=(V \times W) \cap \mathcal{T}, f(a, W)=f(\{a\}, W)$, and $f(V, a)=f(V,\{a\})$. If we regard a mapping $f: \mathcal{T} \rightarrow \mathbb{R}^{\ell}$ as a contingent transfer, then $f(a, W)$ is the total of commodities that are sent from agent $a$ to agents belonging to $W$, and $f(V, a)$ is the total of commodities that are sent to agent $a$ from agents in $V$. (See fig. 4.)

Given a transfer network economy $\mathcal{E}$ and a stochastic transfer $\tau=\left(\tau_{1}, \tau_{2}\right)$, it is assumed that the consumptions take place after second round transfers are completed. Let us denote by $\tau^{s}(\omega, t)$ the total of commodities in state $\omega$ sent from $t_{1}$ to $t_{2}$ where $t=\left(t_{1}, t_{2}\right)$, i.e.,

$$
\tau^{s}(\omega, t) \equiv \tau_{1}(\omega, t)+\tau_{2}(\omega, t)
$$

and by $\tau^{\sigma}(\omega, t)$, the total of commodities in state $\omega$ sent from $t_{1}$ to $t_{2}$ and actually received by $t_{2}$, i.e.,

$$
\tau^{\sigma}(\omega, t) \equiv \sigma_{1}(\omega, t) \tau_{1}(\omega, t)+\sigma_{2}(\omega, t) \tau_{2}(\omega, t)
$$

Thus, by our notational convention, $\tau^{s}(\omega, a, A)$ represents the total of commodities sent by $a \in A$ to other agents in $A$ and $\tau^{\sigma}(\omega, A, a)$ represents the total of commodities sent by 
various agents in $A$ that are received by $a$. We say that a stochastic transfer $\tau$ is feasible for $\mathcal{E}$ if for every $a \in A$ and $\omega \in \Omega$, we have

$$
0 \leq \tau^{s}(\omega, a, A) \leq e(a)
$$

\subsection{Consumptions resulting from a stochastic transfer}

Let $\tau$ be a feasible stochastic transfer for $\mathcal{E}$. We now provide an explicit definition of agents' consumptions resulting from a stochastic transfer. Each agent $a$ is endowed with a bundle $e(a)$ of various commodities to start out with. Out of what $a$ has, $a$ transfers a bundle $\tau^{s}(\omega, a, A)$ comprising of various commodities to agents in $A$ and receives a commodity bundle $\tau^{\sigma}(\omega, A, a)$. In general one has

$$
\sum_{a \in A} \tau^{\sigma}(\omega, A, a)<\sum_{a \in A} \tau^{s}(\omega, a, A)
$$

for a.e. $\omega \in \Omega$ (that is, the total of commodities received by various agents are strictly less than that of commodities sent out by the agents as first round transfers are risky and second round transfers are costly.) Thus, after the stochastic transfer $\tau$ is completed, the amount of commodities at hand for agent $a$, which is available for $a$ 's consumption, is given by

$$
e(a)-\tau^{s}(\omega, a, A)+\tau^{\sigma}(\omega, A, a)
$$

\section{Efficiency and Competition}

\subsection{Efficiency}

Given a stochastic transfer $\tau$ for $\mathcal{E}$ and a nonempty subset $C \subset A$, we say $\tau$ is $C$-inefficient if there is another stochastic transfer $\mu$ feasible for $\mathcal{E}$ such that

$$
\begin{gathered}
(1) \quad(\forall a \in C) E\left[u_{a}\left(e(a)-\mu^{s}(\omega, a, A)+\mu^{\sigma}(\omega, A, a)\right)\right] \\
\geq E\left[u_{a}\left(e(a)-\tau^{s}(\omega, a, A)+\tau^{\sigma}(\omega, A, a)\right)\right], \\
(2) \quad(\exists a \in C) E\left[u_{a}\left(e(a)-\mu^{s}(\omega, a, A)+\mu^{\sigma}(\omega, A, a)\right)\right] \\
>E\left[u_{a}\left(e(a)-\tau^{s}(\omega, a, A)+\tau^{\sigma}(\omega, A, a)\right)\right], \\
(3) \quad(\text { for } a . e . \omega \in \Omega)\left(\forall t=\left(t_{1}, t_{2}\right) \in \mathcal{T} \text { with } t_{1} \notin C \text { or } t_{2} \notin C\right) \\
\mu_{1}(\omega, t)=\tau_{1}(\omega, t) \quad \text { and } \quad \mu_{2}(\omega, t)=\tau_{2}(\omega, t) .
\end{gathered}
$$

$\tau$ is $C$-efficient if it is not $C$-inefficient. A feasible stochastic transfer $\tau$ is Pareto efficient if it is $A$-efficient. A feasible stochastic transfer $\tau$ is totally efficient if for any nonempty $C \subset A$, it is $C$-efficient. 


\subsection{Competition}

Since we are concerned with efficiency and competitiveness properties of a network transfer economy without explicit consideration of "market prices", we appeal to the game theoretic notion of core. For a nonempty $C \subset A$, a stochastic transfer $\tau$ is $C$-subcompetitive (or $C$-dominated) if there is another feasible stochastic transfer $\mu$ satisfying (1) and (2) above and

$$
\begin{gathered}
\left(3^{\prime}\right) \quad(\text { for a.e. } \omega \in \Omega)\left(\forall t=\left(t_{1}, t_{2}\right) \in \mathcal{T} \text { with } t_{1} \notin C \text { or } t_{2} \notin C\right) \\
\mu_{1}(\omega, t)=0 \quad \text { and } \quad \mu_{2}(\omega, t)=0 .
\end{gathered}
$$

A feasible stochastic transfer $\tau$ is said to be $C$-competitive if it is not $C$-subcompetitive. Finally, a stochastic transfer $\tau$ is called totally competitive if it is $C$-competitive for all nonempty $C \subset A$. In particular, $\tau$ is called individually rational if it is $\{a\}$-competitive for all $a \in A$.

\section{$3.3 \quad$ Facts}

One can check the following lemma and propositions without difficulty.

Lemma 1 Assume $C_{1} \subset C_{2}$. If $\tau$ is $C_{2}$-efficient, then it is $C_{1}$-efficient.

Proof Let $C_{1} \subset C_{2}$. Assume $\tau$ is $C_{2}$-efficient but not $C_{1}$-efficient. Then, there is another feasible stochastic transfer $\mu$ satisfying the properties (1), (2) and (3) with $C=C_{1}$. Since $C_{1} \subset C_{2}$, it also implies that $\tau$ is $C_{2}$-inefficient contradicting to the assumption.

Proposition $1 \tau$ is totally efficient if and only if it is Pareto efficient.

Proof Assume $\tau$ is totally efficient. Then, it is trivially $A$-efficient.

On the other hand, assume $\tau$ is Pareto-efficient or $A$-efficient. Then, by Lemma 1 , for any $C \subset A, \tau$ is $C$-efficient. Thus, it is totally efficient.

Proposition 2 If $\tau$ is totally competitive, then it is totally efficient.

Proof Let $\tau$ be totally competitive. Assume $\tau$ is not $A$-efficient. Then, there is another feasible stochastic transfer $\mu$ satisfying the properties (1),(2) and (3) with $C=A$. Since the complement of $A$ is empty, $\tau$ must be $A$-subcompetitive contradicting the fact that it is totally competitive. Thus, it must be $A$-efficient. It follows from Proposition 1 that $\tau$ is totally efficient. 


\subsection{A Speci fic Result}

At this point we would like to introduce a network transfer economy with a very specific directed graph which has relevance to some economic problems. (See, for example, [2] and [3].)

The structure of a specific directed graph that we consider here is what we call a "Wicksell triangle" plus a simple barter which is given by the following (see fig. 5) :

- $A=\left\{a_{1}, a_{2}, a_{3}, a_{4}\right\}$

- $\mathcal{T}=\left\{\left(a_{1}, a_{2}\right),\left(a_{2}, a_{3}\right),\left(a_{3}, a_{1}\right),\left(a_{3}, a_{4}\right),\left(a_{4}, a_{3}\right),\left(a_{1}, a_{1}\right),\left(a_{2}, a_{2}\right),\left(a_{3}, a_{3}\right),\left(a_{4}, a_{4}\right)\right\}$

For a transfer network economy with a directed graph which is given by a Wicksell triangle plus a simple barter, we have the following characterization of its total competitiveness.

Proposition 3 Let $\mathcal{E}=\left\{(A, \mathcal{T}), e,\left(u_{a}\right)_{a \in A}, \sigma, \mathcal{P}\right\}$ be a transfer network economy with a directed graph $(A, \mathcal{T})$ as specified above. Assume each agent's utility function $u_{a}, a \in A$, be locally nonsatiated in his own endowment goods. Let $\tau$ be a stochastic transfer feasible for $\mathcal{E}$. Then, $\tau$ is totally competitive if and only if the following conditions hold: $\tau$ is individually rational, totally efficient, $\left\{a_{1}, a_{2}, a_{3}\right\}$ - and $\left\{a_{3}, a_{4}\right\}$-competitive.

Proof Assume $\tau$ is totally competitive. Then, it must satisfy the conditions by definition and proposition 2 .

Conversely, suppose that a stochastic transfer $\tau$ is individually rational, totally efficient, $\left\{a_{1}, a_{2}, a_{3}\right\}$ - and $\left\{a_{3}, a_{4}\right\}$-competitive. The only nonemty subset $C$ for which $\tau$ could be $C$-subcompetitive without explicitly violating one of the three conditions are then:

- A. The subsets consisting of $a_{4}$ together with either $a_{1}$ or $a_{2}$; and

- B. The subsets to which exactly two members of $\left\{a_{1}, a_{2}, a_{3}\right\}$ belong.

Call these type A and type B subsets respectively. We now show that any individually rational and totally efficient stochastic transfer $\tau$ cannot be $C$-subcompetitive for any subset of either type A or type B.

Assume that a stochastic transfer $\mu$ satisfied the conditions (1), (2), and (3') for a subset $C$. Suppose $C$ is of type A. Since $\tau$ is totally efficient, it is $C$-efficient and thus the only possible $\mu$ satisfying the conditions (1) and (3') is autarky, i.e., $\mu^{s}(\omega, a, A)=0$ for $a \in A$ when $C$ is $\left\{a_{1}, a_{4}\right\}$ or $\left\{a_{2}, a_{4}\right\}$. But since $\tau$ is individually rational, $\mu$ cannot satisfy condition (2) for either of those two subsets.

Now suppose that $C$ is of type B. That is, either $C=\{a, b\} \subset\left\{a_{1}, a_{2}, a_{3}\right\}$, or else $C=D \cup\left\{a_{4}\right\}$ and $D=\{a, b\} \subset\left\{a_{1}, a_{2}, a_{3}\right\}$. In the former case, without loss of generality, $a=a_{j}, b=a_{i}$ with $j=i+1(\bmod 3)$. The only transfer that can occur in any state of 


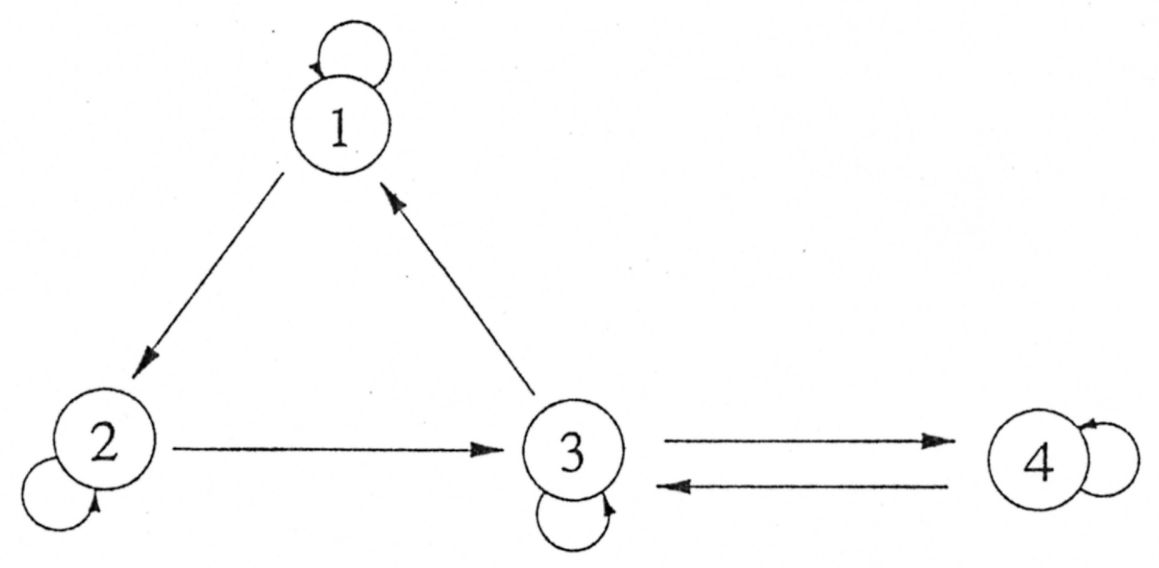

Fig. 5 A Wicksell Triangle pius a Simple Barter 
nature between these two agents is for $a$ to receive some of $b$ 's endowment goods. This must happen with positive probability, in order for (2) to be satisfied for $a$. In that case, though, neither (1) nor (2) can be satisfied for $b$. The same argument applies in the latter case, unless $b$ is agent $a_{3}$ who receives some of the endowment goods of agent $a_{4}$ (and $a$ is agent $\left.a_{1}\right)$. If so, define a stochastic transfer $\theta=\left(\theta_{1}, \theta_{2}\right)$ by

$$
\theta_{n}(\omega, t)= \begin{cases}0 & \text { if } t=\left(a_{3}, a_{1}\right) \\ \mu_{n}(\omega, t) & \text { otherwise }\end{cases}
$$

for $n=1,2$. Agent $a_{3}$ strictly prefers $\theta$ to $\mu$, and agent $a_{4}$ is indifferent between $\theta$ and $\mu$. Therefore, if $\tau$ were $C$-subcompetitive for $\mu$, then $\tau$ would be $\left\{a_{3}, a_{4}\right\}$-subcompetitive for $\theta$, contrary to hypothesis.

\subsection{Decomposition of a graph}

Before proceeding to obtain further results on efficiency and competition, we would like to discuss concepts concerning the decomposition of a graph.

Let $(A, \mathcal{T})$ be a directed graph. $\left\{\left(A_{i}, \mathcal{T}_{i}\right)\right\}_{i=1, \cdots, n}$ is a decomposition of $(A, \mathcal{T})$ if $\left\{A_{i}\right\}_{i=1, \cdots, n}$ is a partition of the set $A$ and $\mathcal{T}_{i}=\mathcal{T} \cap\left(A_{i} \times A_{i}\right)$. We write $\mathcal{T}_{i j}=\mathcal{T} \cap\left(A_{i} \times A_{j}\right)$, and put $\mathcal{T}_{i j}^{-1} \equiv\left\{(a, b) \in A_{i} \times A_{j} \mid(b, a) \in \mathcal{T}_{j i}\right\}$ for $i \neq j$. A decomposition $\left\{\left(A_{i}, \mathcal{T}_{i}\right)\right\}_{i=1, \cdots, n}$ of a directed graph is called perfect if $\mathcal{T}_{i j}=\emptyset$ for all $i \neq j$.

Let $\mathcal{E}=\left\{(A, \mathcal{T}), e,\left(u_{a}\right)_{a \in A}, \sigma, \mathcal{P}\right\}$ be a transfer network economy. A feasible stochastic transfer $\tau$ is said to be $C$-totally competitive if it is $C^{\prime}$-competitive for all nonempty $C^{\prime} \subset C$.

We now state a very easy result.

Proposition 4 Let $\mathcal{E}=\left\{(A, \mathcal{T}), e,\left(u_{a}\right)_{a \in A}, \sigma, \mathcal{P}\right\}$ be a transfer network economy. Let $\left\{\left(A_{i}, \mathcal{T}_{i}\right)\right\}_{i=1, \cdots, n}$ be a perfect decomposition of $(A, \mathcal{T})$. Then, a feasible stochastic transfer $\tau$ is totally competitive if and only if it is $A_{i}$-totally competitive for each $i=1, \cdots, n$.

Proof Let $\left\{\left(A_{i}, \mathcal{T}_{i}\right)\right\}_{i=1, \cdots, n}$ be a perfect decomposition of the directed graph $(A, \mathcal{T})$. If $\tau$ is a feasible stochastic transfer which is totally competitive, then it is trivially $A_{i}$-totally competitive for all $i=1, \cdots, n$.

Conversely, assume that $\tau$ is $A_{i}$-totally competitive for each $i=1, \cdots, n$. Assume that there existed a nonempty subset $C \subset A$ and a feasible stochastic transfer $\mu$ such that $\tau$ is not $C$-competitive with respect to $\mu$. Then, there exists $j \in\{1, \cdots, n\}$ such that some of the agents in $C \cap A_{j}$ strictly prefer $\mu$ to $\tau$. Define a feasible stochastic transfer $\theta$ to be identical with $\mu$ for agents in $C \cap A_{j}$. (which is possible as the decomposition is perfect), and $\theta$ to be 0 outside $C \cap A_{j}$. Then, $\tau$ becomes $C \cap A_{j}$-subcompetitive under $\theta$, contradicting the fact that $\tau$ is $A_{j}$-totally competitive. Therefore, $\tau$ must be totally competitive. 
The proposition 4 shows that when a transfer network economy is decomposed into independent parts, the competitiveness of the entire part simply reduces to the competitiveness of each independent part. This simple result motivates us to investigate circumstances under which the competitiveness of each part is equivalent to that of the entire economy even when the entire graph cannot be decomposed in a perfect manner. It turns out that if each decomposed part along with its linkage between the decomposed parts is competitive, then the competitiveness of the entire part is obtained.

Given a directed graph $(A, \mathcal{T})$, a subset $C$ is a path from $a_{1}$ to $a_{n}$ if $C=\left\{a_{1}, a_{2}, \cdots, a_{n}\right\}$ with $\left(a_{i}, a_{i+1}\right) \in \mathcal{T}$ for $i=1, \cdots, n-1$. If all $a_{1}, \cdots, a_{n}$ are distinct, it is called a strict path. A path $C$ from $a_{1}$ to $a_{n}$ is a cycle if $a_{1}=a_{n}$ and if $\left\{a_{1}, a_{2}, \cdots, a_{n-1}\right\}$ is a strict path. In this case we also say that $C$ is a cycle into $a_{1}$ or $a_{n}$. A path $C$ from $a_{1}$ to $a_{n}$ or a cycle into $a_{1}=a_{n}$ is called unilateral if $\left(a_{i+1}, a_{i}\right) \notin \mathcal{T}$ for all $i=1, \cdots, n-1$. It is called bilateral or a barter if $\left(a_{i+1}, a_{i}\right) \in \mathcal{T}$ for all $i=1, \cdots, n-1$.

Let $\left\{\left(A_{i}, \mathcal{T}_{i}\right)\right\}_{i=1, \cdots, n}$ be a decomposition of a directed graph $(A, \mathcal{T})$. Define two subsets $B_{i j}$ and $L_{i j}$ of $A_{i}$ for each $i=1, \cdots, n$.

$$
\begin{aligned}
& L_{i j} \equiv\left\{a \in A_{i} \mid\left(\exists b \in A_{j}\right) \quad(a, b) \in \mathcal{T}_{i j}\right\}, \\
& B_{i j} \equiv\left\{a \in A_{i} \mid\left(\exists b \in A_{j}\right) \quad(a, b) \in \mathcal{T}_{i j} \cap \mathcal{T}_{i j}^{-1}\right\} .
\end{aligned}
$$

An element a in $L_{i j}$ will be called a transfer linkage of $A_{i}$ with respect to $A_{j}$. Similarly, an element $a$ in $B_{i j}$ will be called a barter linkage of $A_{i}$ with respect to $A_{j}$. If, for any given $i, j=1, \cdots, j, a \in A_{i}, b \in A_{j}$, there always exists a path from $a$ to $b$, then the directed graph is said to be irreducible.

We would like to extend proposition 4 to the case of a transfer network with an irreducible directed graph. This will be done in a manner corresponding to proposition 3 where the directed graph is decomposed into two parts that has a barter linkage. We now state a proposition which extends both proposition 3 and proposition 4 when the directed graph is decomposed into two parts. Due to the irreducibility of the entire network of the graph, the requirement of the competitiveness of decomposed parts alone is not enough.

Proposition 5 Let $\mathcal{E}=\left\{(A, \mathcal{T}), e,\left(u_{a}\right)_{a \in A}, \sigma, \mathcal{P}\right\}$ be a transfer network economy and $\left\{\left(A_{i}, \mathcal{T}_{i}\right)\right\}_{i=1,2}$ be a decomposition of $(A, \mathcal{T})$ such that each $A_{i}, i=1,2$, is a unilateral cycle and $L_{i j}=B_{i j}$ for each $i, j=1,2, i \neq j$. Assume each agent's utility function $u_{a}, a \in A$, be locally nonsatiated. Let $\tau$ be a feasible stochastic transfer. Then, $\tau$ is totally competitive if and only if the following conditions hold: $\tau$ is totally efficient, $A_{i} \cup B_{j i}$-totally competitive for each $i, j=1,2, i \neq j$, and $B_{12} \cup B_{21}$-competitive.

Proof Assume $\tau$ is totally competitive. Then, it must satisfy the conditions by definition and proposition 2.

Conversely, suppose that the decomposition $\left\{\left(A_{i}, \mathcal{T}_{i}\right)\right\}_{i=1,2}$ is such that each $A_{i}$ is a unilateral cycle. Assume $\tau$ is totally efficient, $A_{i} \cup B_{j i}$-totally competitive for each $i, j=$ 
$1,2, i \neq j$, and $B_{12} \cup B_{21}$-competitive. Suppose there existed another feasible stochastic transfer $\mu$ for which $\tau$ were $C$-subcompetitive for some nonempty $C \subset A$.

Since $\tau$ is $A_{1}$-and $A_{2}$-totally competitive, one must have $C \cap A_{i} \neq \emptyset$ for each $i$ and $B_{i j} \cap C \neq \emptyset$ for each $i, j=1,2, i \neq j$. Put $C_{i}=C \cap A_{i}$ and $C_{i j}=B_{i j} \cap C$ for each $i, j=1,2, i \neq j$. As $\tau$ is totally efficient, we must have $C_{i} \neq A_{i}$ for some $i$. Consider the following two possible cases.

- Case 1: $C_{i} \neq A_{i}$ for each $i$.

Since $A_{i}$ is a unilateral cycle, $C_{i}$ is not a cycle and hence, for each $i$, there is $a_{i}^{*}$ who cannot receive any commodities from other agents under the transfer $\mu$. Thus, under $\mu$, no agents in $C_{i} \backslash C_{i j}$ can transfer endowment goods to other agents without violating the individual rationality. This means that only agents who would strictly prefer $\mu$ over $\tau$ are restricted to those belonging to $C_{12} \cup C_{21}$ only. Since agents in $C_{i} \backslash C_{i j}$ just consume their own endowment goods under $\mu$, it follows that $\tau$ is $C_{12} \cup C_{21}$-subcompetitive with respect to $\mu$, contradicting to the hypothesis.

- Case 2: $C_{i}=A_{i}$ for some $i$.

Without loss of generality, assume $C_{1}=A_{1}$ and $C_{2} \neq A_{2}$. Since $A_{2}$ is a unilateral cycle, the agents in $C_{2} \backslash C_{21}$ must consume only their endowments goods under the transfer $\mu$ as $\tau$ is individually rational. Therefore, only agents who would strictly prefer $\mu$ over $\tau$ are restricted to those belonging to $A_{1} \cup C_{21}$ only. It follows that $\tau$ is $A_{1} \cup C_{21}$-subcompetitive with respect to $\mu$, contradicting to the hypothesis.

We thus proved that $\tau$ must be totally competitive.

The above proposition 5 essentially shows that even when the entire graph cannot be decomposed in a perfect manner, when a transfer network economy is decomposed into parts, the competitiveness of the entire part reduces to the competitiveness wihtin each decomposed part along with its barter linkage between the decomposed parts.

\section{Competitive Stochastic Transfers in the Wicksell Triangle plus a Simple Barter}

\subsection{A further speci fication}

Given a transfer network economy $\mathcal{E}=\left\{(A, \mathcal{T}), e,\left(u_{a}\right)_{a \in A}, \sigma, \mathcal{P}\right\}$, we are interested in giving a more specific characterization of totally competitive stochastic transfers. For this purpose, we shall go back to a very specific model of the Wicksell triangle plus a simple barter which was presented in the subsection 3.4. Thus, throughout this section we take the directed graph to be given by the following $(A, \mathcal{T})$ : 
- $A=\left\{a_{1}, a_{2}, a_{3}, a_{4}\right\}$

- $\mathcal{T}=\left\{\left(a_{1}, a_{2}\right),\left(a_{2}, a_{3}\right),\left(a_{3}, a_{1}\right),\left(a_{3}, a_{4}\right),\left(a_{4}, a_{3}\right),\left(a_{1}, a_{1}\right),\left(a_{2}, a_{2}\right),\left(a_{3}, a_{3}\right),\left(a_{4}, a_{4}\right)\right\}$

The transfer network economy $\mathcal{E}=\left\{(A, \mathcal{T}), e,\left(u_{a}\right)_{a \in A}, \sigma, \mathcal{P}\right\}$ is further specified by letting:

- $a_{i}=i, \quad i=1, \ldots, 4$

- $L=R^{4}$

- $e(i)=(0, \ldots, \stackrel{i}{1}, \ldots, 0)$

- $\mathcal{P}_{1}=\{\Omega\}, \mathcal{P}_{2}=\{S, F\}, \operatorname{Pr}(S)=\bar{\sigma}$

- Transfer technology $\sigma=\left(\sigma_{1}, \sigma_{2}\right)$ is given by:

- For $t=(2,3), \quad(\forall \omega \in \Omega) \sigma_{1}(\omega, t)=\chi_{S}(\omega)$.

- For all other $t \in \mathrm{T},(\forall \omega \in \Omega) \sigma_{1}(\omega, t)=1$.

- For all $t \in \mathrm{T},(\forall \omega \in \Omega) \sigma_{2}(\omega, t)=\rho$.

$-1>\bar{\sigma}>\rho \geq \frac{1}{2}$

- We specify the agents' utilities as follows.

$$
\begin{aligned}
u_{1}(x)= & \ln \left(x_{1}+\beta x_{3}\right) \\
u_{2}(x)= & \ln \left(x_{2}+\beta x_{1}\right) \\
u_{3}(x)= & \ln \left(x_{3}+\beta x_{2}+\not{ }_{4}\right) \\
u_{4}(x)= & \ln \left(x_{4}+\varphi x_{3}\right) \\
& \quad \text { with } \beta>\max \left\{\bar{\sigma}^{-1}, \rho^{-1}\right\}, 0<\varphi \psi<1 .
\end{aligned}
$$

Here, goods received in trade are "better" substitutes for endowment goods for essential participants 1,2,3. Agent 4 considers agent 3's good to be a "worse" substitute for his own endowment good, and agent 3 considers 4's good to be a "worse" substitute for agent 2's good or even for his own endowment good.

\subsection{Interpretation}

Motivation of introducing a model described in the previous subsection 4.1 is discussed in [2]. Here, let us give a brief description of an interpretation of the model in an economic context.

It is a model of a network or an arrangement of transactions that involve a risky transfer of assets. Transactions are generated endogenously. There is a risk in asset transfers and 
one is concerned with the question of optimal risk management in such a network. Assets may well be usual commodities and not limited to financial assets. Some specific questions regarding risk management in a network of transactions are the following. If there is some risk of failure in a transfer from one party to another, should the transfer be done through that arrangement? If so, then what considerations are relevant to determining whether third parties ought to share that risk? Are there conditions under which the general public or the government (in the case of a private arrangement) ought to bear some risk and, if so, what level of compensation would it be appropriate for them to receive? One can address these questions by analyzing a schematic, formal, model of a stochastic transfer introduced above. The particular model of the Wicksell triangle plus a simple barter has been motivated as a simplest model to analyze the questions posed.

Suppose that our task is to formulate a model of a transaction that involves a risky asset transfer. The model should be rich enough to describe such a transaction recognizably, but simple enough to be analytically tractable. Consider what sort of model could satisfy both the requirements of richness and simplicity. A transaction is a related set of asset transfers between agents. An asset transfer involves two agents, the donor and the recipient, but a transaction can generally involve more than two agents. Therefore, at the very least, a model of a transaction involving a risky transfer should include three agents, so that a distinction can be drawn between a participant in the broad transaction and a participant (that is, the donor or the recipient) in the specific transfer where the risk occurs. In order for the third-party participant in the transaction - that is, the participant who is neither the donor nor the recipient of the risky transfer - to be essential to making a mutually beneficial transaction, there should be no "double coincidence of wants" between the donor and the receiver. This consideration suggests modeling the three participants as a "Wicksell triangle."

There is a distinction between two types of third party (or potential third party) that a model ought to capture. A third party to risky transfer in a Wicksell triangle might be intrinsically necessary in the sense that the donor and recipient of the risky transfer would have no double coincidence of wants, even if the transfer did not involve risk. For characterizing the differences between the roles of these two types of third parties, a fourtrader model (including both an intrinsic third party and a trader whose only involvement would be to share risk) can be useful. On the basis of these considerations, one is lead to the four-agents model of the Wicksell triangle plus a simple barter, where agent 1 is assumed to be essential to a mutually beneficial transaction but that agent 2 is the donor and agent 3 is the recipient of the risky transfer. The attributes of agent 4 , who constitutes to be the potential partner of the essential participants, is specified in such a way that agent 4 can only participate in a risk-sharing capacity. This should be clearly seen from the specification of the agents' utilities in (4.3). 


\subsection{Some notation}

Given a stochastic transfer $\tau$, we shall write for convenience

$$
\begin{aligned}
\tau_{1}\left(\omega, t_{1}, t_{2}\right)= & \begin{cases}\tau_{i}^{1}, i=t_{1} & \text { for } t_{1}=1,2,4 \\
\tau_{3}^{1} & \text { for } t_{1}=3, t_{2}=1 \\
\tau_{5}^{1} & \text { for } t_{1}=3, t_{2}=4\end{cases} \\
& \text { for all } \omega \in \Omega,
\end{aligned}
$$

When a stochastic transfer $\tau$ is clear from the context, we may write for simplicity

$$
\begin{aligned}
& C_{i}^{S}=1-\tau_{i}^{1}-\tau_{i}^{S}+a_{i-1}\left(\tau_{i-1}^{1}+\rho \tau_{i-1}^{S}\right) \\
& C_{i}^{F}=1-\tau_{i}^{1}-\tau_{i}^{F}+a_{i-1}\left(\tau_{i-1}^{1}+\rho \tau_{i-1}^{F}\right)
\end{aligned}
$$

for $i=1,2,4$ where $i-1=3$ for $i=1$ and $i-1=5$ for $i=4$, and $a_{i}=\beta$ for $i=1,2,3$, $a_{4}=\gamma$, and $a_{5}=\varphi$. For $i=3$, we have

$$
\begin{aligned}
& C_{3}^{S}=1-\tau_{3}^{1}-\tau_{3}^{S}+\beta\left(\tau_{2}^{1}+\rho \tau_{2}^{S}\right)-\tau_{5}^{1}-\tau_{5}^{S}+\gamma\left(\tau_{4}^{1}+\rho \tau_{4}^{S}\right) \\
& C_{3}^{F}=1-\tau_{3}^{1}-\tau_{3}^{F}+\beta \rho \tau_{2}^{F}-\tau_{5}^{1}-\tau_{5}^{F}+\gamma\left(\tau_{4}^{1}+\rho \tau_{4}^{F}\right)
\end{aligned}
$$

$C_{i}^{E}$ is interpreted as "real" consumption level of agent $i$ in event $E$ in the sense that it directly determines $i$ 's utility level in event $E \in \mathcal{P}_{2}$.

Given a stochastic transfer $\mu$, a net transfer gap of agent $i$ is defined by:

$$
g_{i}(\mu)=1-\mu_{i}^{1}-\chi_{\{3\}} \mu_{5}^{1}-\max \left\{\mu_{i}^{S}+\chi_{\{3\}} \mu_{5}^{S}, \mu_{i}^{F}+\chi_{\{3\}} \mu_{5}^{F}\right\} .
$$

It represents the maximal amount that agent $i$ can further transfer to other agents. Here, $\chi_{\{3\}}=1$ for $i=3$ and $=0$ otherwise.

We may view the Wicksell triangle as a transfer system, in which case we may refer to the agents belonging to the Wicksell triangle as essential participants to the system and agent 4 as the inessential participant. Then, the amount $g_{i}(\mu)$ is the maximal amount that agent $i$ can further transfer to other agents including the inessential participant. Hence, if we define transfer gap $\bar{g}_{i}(\mu)$ by setting

- $\bar{g}_{i}(\mu)=g_{i}(\mu)$ for $i=1,2$, and

- $\bar{g}_{3}(\mu)=1-\mu_{3}^{1}-\max \left\{\mu_{3}^{S}, \mu_{3}^{F}\right\}$, 
then $\bar{g}_{i}$ 's represent the maximal amount that agent $i$ can transfer to other agents among essential participants.

\subsection{Assumption on parameter values}

We shall require conditions which guarantee to generate nontrivial stochastic transfer in the given transfer network economy $\mathcal{E}=\left\{(A, \mathcal{T}), e,\left(u_{a}\right)_{a \in A}, \sigma, \mathcal{P}\right\}$. We assume the following conditions on parameter values:

- $\varphi \gamma>\left(\frac{1-\bar{\sigma}}{\rho}\right) \frac{1}{1-\rho}$, and

- $\beta \rho>\sqrt[3]{2}, \rho>\sqrt[3]{2} / 2$.

The first inequality is satisfied, if, for example, $\varphi \gamma>0.63$ when $\bar{\sigma}=0.9$ and $\rho=0.8$. It is also satisfied whenever $\varphi \gamma>r^{\bar{\sigma} \rho}$ where

$$
r^{\bar{\sigma} \rho} \equiv\left(\frac{1-\bar{\sigma}}{\bar{\sigma}}\right)\left(\frac{\rho}{1-\rho}\right) .
$$

The second and the third inequalities are to ensure that second round transfers are not too costly to make such transfers.

\subsection{Speci fic results}

Finally, let us present two specific results concerning the efficiency and optimality properties of stochastic transfers in the model of the present section which have been proved elsewhere (see, e.g., [2]). These properties are stated in terms of total competitiveness of transfers.

Proposition 6 A totally competitive stochastic transfer $\mu$ always specifies state contingent transfers. A typical totally competitive stochastic transfer $\mu$ satisfies:

$$
\begin{aligned}
& \mu_{i}^{1}>0 \quad \text { for } i=1,2,3,5, \mu_{4}^{1}=0, \\
& \mu_{1}^{F}>0, \mu_{2}^{F}>0, \mu_{3}^{F}>0, \mu_{4}^{F}>0, \mu_{5}^{F}=0, \\
& \mu_{1}^{S}=\mu_{3}^{S}=\mu_{4}^{S}=\mu_{5}^{S}=0, \mu_{2}^{S}>0,
\end{aligned}
$$


in which case we have:

$$
\begin{aligned}
\frac{C_{2}^{F}}{C_{2}^{S}}= & \frac{C_{3}^{F}}{C_{3}^{S}}=r^{\bar{\sigma} \rho} \\
\frac{C_{1}^{F}}{C_{1}^{S}} \geq & \left(\frac{1-\bar{\sigma}}{\bar{\sigma}}\right) \frac{1}{1-\rho}>r^{\bar{\sigma} \rho} \\
& \text { with equality holding when } g_{1}(\mu)>0 \\
\frac{C_{4}^{F}}{C_{4}^{S}}= & \frac{1-\bar{\sigma}}{\bar{\sigma}}\left(\frac{1}{\varphi \gamma \rho(1-\rho)}-1\right)>\left(\frac{1-\bar{\sigma}}{\bar{\sigma}}\right) \frac{1}{1-\rho} .
\end{aligned}
$$

One may note the extent to which agents' consumptions that a typical totally competitive stochastic transfer induces are state contingent. Given a typical totally competitive stochastic transfer as in the beginning of the statement of the proposition above, for agent 2 and agent 3 the consumption level in event $F$ relative to that in event $S$ is $r^{\bar{\sigma} \rho}$, which is less than 1 but approaches 1 as the value of $\rho$ becomes closer to $\bar{\sigma}$. This may be interpreted to say that the failure of receipt by agent 3 is compensated by other agents by the factor of $(\rho /(1-\rho))-1$. Agent 2 is as responsible as agent 3 for the loss as his relative consumption level in event $F$ is reduced to the level of agent 3. Agent 1 in turn compensates agent 2 but extent to which he joins in the compensation is less than that of agent 2 so that his relative consumption in event $F$ exceeds $r^{\bar{\sigma} \rho}$. It is very instructive to note that agent 4 also participates in this compensation scheme but extent to which he does compensate agent 3 is much less than those of other agents in the sense that his relative consumption level in event $F$ is higher than those of all the essential participants.

Proposition 7 Let $\mu$ be a totally competitive stochastic transfer. Then:

1. At least one essential participant must be sending all his endowment to other agents in some event. That is,

$$
(\exists i \in\{1,2,3\}) g_{i}(\mu)=0 .
$$

2. Suppose that agent 3 is not sending all of his endowment to other essential participants so that $\bar{g}_{3}(\mu)>0$. Then, $\mu$ is a totally competitive stochastic transfer if and only if agent 3 is making a transfer to agent 4 either by the amount of his transfer gap or by the amount of "feasibility bound" given by

$$
v(\varphi \gamma, \bar{\sigma}, \rho, \beta)=\frac{(1-\bar{\sigma})(1-\varphi \gamma \rho(1-\rho))}{\beta[\varphi \gamma \rho(1-\rho)-(1-\bar{\sigma})]},
$$

whichever is smaller, i.e.,

$$
\mu_{5}^{1}=\min \left\{\bar{g}_{3}(\mu), v(\varphi \gamma, \bar{\sigma}, \rho, \beta)\right\},
$$


and agent 4 in turn is making a state contingent transfer in event $F$ at most the amount given by

$$
\tau_{4}^{F}=\left(\frac{\varphi \gamma \rho(1-\rho)-(1-\bar{\sigma})}{\bar{\sigma} \varphi \gamma \rho(1-\rho)}\right)\left(1+\beta \tau_{5}^{1}\right)
$$

The first part of the proposition 7 is due to our specification of preferences of essential participants that they prefer the endowment of another agent to his own. The second part results from two factors. One is that a totally competitive stochastic transfer in general specifies positive second round transfers in both events $F$ and $S$ as well as a positive first round state non-contingent transfer from agent 2 to agent 3. This ensures agent 3's consumption in event $F$ relative to that in event $S, C_{3}^{F} / C_{3}^{S}$, to be given by $r^{\bar{\sigma} \rho}$. Second is that under this circumstance, the expected utility of both of the agents 3 and 4 can be increased whenever first round state non-contingent transfer from 3 to 4 and second round state contingent transfer in event $F$ from 4 to 3 can be increased. For a first round state non-contingent transfer from 3 to $4, \tau_{5}^{1}$, the maximal amount that agent 4 would be just willing to send to 3 is given by the amount shown in (4.6). For details, one is referred to $[2]$.

\section{References}

[1] Allen, Beth, "Supermechanisms," Department of Economics, University of Minnesota, 1993.

[2] Fujiki, Hiroshi, Edward J. Green, and Akira Yamazaki, "Sharing the Risk of Settlement Failure," mimeo., October 1998.

[3] Fujiki, Hiroshi, Edward J. Green, and Akira Yamazaki, "Sharing the Risk of Settlement Failure:Synopsis," Proceedings of International Conference on Risk Management and Systemic Risk, Bank of Japan, March 1999. 